\title{
Inviting teachers to use educational robotics to foster mathematical problem-solving
}

\author{
Vladimir Estivill-Castro ${ }^{1}$ \\ Griffith University, Nathan 4111, Australia, \\ v.estivill-castro@griffith.edu.au,
}

\begin{abstract}
We have developed three lessons supported by the principles of inquiry-based learning (IBL) and problem-based learning (PBL) in educational robotics with the aim of steering and emphasising the mathematics aspects of the curriculum and the role of mathematics in STEM, while also touching on the social context and impact of STEM. Our goal is to inspire and prompt the curiosity in the participants to seek further understanding in mathematics, to develop mathematical thinking and problem-solving skills, and to see applicability in the emerging world where artificial intelligence and automation are transforming the skills learners will use as professionals. Moreover, we have delivered our ideas to educators in high-school who indicated they would incorporate our challenges and tools to cross-pollinate different areas of STEM.
\end{abstract}

\section{Introduction}

As part of our university's outreach activities, and as others previously $[18,38$, 31], we aim at establishing motivating links with the STEM curriculum using hands-on experiences with robots. More importantly, we hope to inspire the so much needed curiosity [15] and assist talented individuals to seek explorations beyond our activities and encourage their teachers to develop lessons around our proposed challenges. At a minimum, we provide some exposure to our participants for the usefulness of mathematical thinking in problem-solving.

The context also derives from the need to ensure high-school pupils meet prerequisites from our programs that offer professional outcomes in STEM. In Queensland, for example, the senior Mathematics (Year-11 and Year-12) curriculum is structured into Maths A, Maths B, and Maths C. Maths A covers more practical topics than Maths B and Math C, and although it is OP eligible (part of the weighted average for ranking for a government-funded place in university), it is insufficient for admission into Software Engineering (or other engineering disciplines) or the Bachelor of Computer Science. At a minimum, candidates require Maths B as it demands more advanced algebra skills than Maths A and more sophisticated mathematical thinking. Maths C goes beyond Maths B, and covers additional pure-maths topics (including complex numbers, matrices, vectors, further calculus and number theory). We note that topics like Control Theory [1] would require to deal with feedback-loop control and core notions of automation need complex numbers, matrices, vectors, calculus and differential equations. Thus, ideally, we aim at inspiring students to pursue Maths C. A study covering 10 years (2007-2016) by the Australian Mathematical Science 
Institute reports that across Australia participation in "Advanced Mathematics (Maths C for Queensland) has steadily dropped from $10.2 \%$ in 2007 to $9.5 \%$ in 2017. A very similar decreasing trend has been recorded for "Intermediate Maths" (Maths B in Queensland): from 21.2\% in 2007 to $19.4 \%$ in 2016 [3].

We have developed 3 lessons supported by the principles of inquiry-based learning (IBL), problem-based learning (PBL) with the aim of steering and emphasising the mathematics aspects of the curriculum and the role of mathematics in STEM, while also touching on the social and context impact of STEM. We hope that topics such as vectors and matrices emerge from the IBL and PBL exploration as useful mathematics modelling tools, and alleviate the stigma that Math C topics are pure-mathematics with little application. Hence, our activities are aimed at the later years of the high-school curriculum and offering opportunities for teachers of those senior years for interaction in the development of high-school lessons or projects involving STEM and using robots. We hope to bridge the teachers from the mathematics department with those of the science departments, those of new STEM initiatives, those on technology and IT.

Our goal is to prompt the curiosity in the participants to seek further understanding in mathematics, to develop mathematical thinking and problem-solving skills, and to see applicability in the emerging world of where artificial intelligence and automation are radically transforming the skills learners will use as professionals. We note that problem-solving (highly regarded as a 21st-century skill [33] and core to STEM skills [12]) cannot be achieved without conceptual understanding, especially for mathematics [40].

In Section 2 we provide some more details regarding the context in which our educational experiences take place. We will provide some information on our chosen infrastructure, and then we will briefly describe our first challenge in Section 3. While our first challenge is perhaps more IBL and less PBL (although IBL is the core framework for PBL [24]), our second challenge is probably more PBL than IBL. We will provide a few more details for the second challenge in Section 4. Because of space we only touch briefly on our third challenge. We have conducted sessions describing the activities to three groups of high-school teachers as part of two professional-development conferences to teachers. We aimed to evaluate whether they would adopt these ideas into more elaborate series of lessons and project. Section 6 will present the results of the survey.

\section{Context}

We regularly receive at the university campus students from high school as part of our STEM educational activities. The students have very different backgrounds because they can be as young as Year 6 ( \pm 12 y.o.), or already in Year 12 . The may have (but maybe not because they are not even in high-school) an initiation to technology, and in particular (coding) programming. But they typically see little value in the mathematics they are studying, or the implications in society by developments in STEM.

Although in some cases using robots was not effective [14], there is now strong evidence that involving learners in robotics, and in particular, robotics 
competitions and informal camps, stimulates interest for STEM [7, 21, 29, 8, 12, $11,22,23]$. For example, interest in STEM professions is increased among middleschool students when the activities are perceived as fun, and involve handson experiences [19]. Some have identified that learning with robots provides a positive social experience, access to an engaged community and feelings of success [16]. Because of this impact, several projects to enhance educational robotics as a catalyst for curiosity in STEM have appeared. In Europe, one example of an educational platform is the Orbital educational framework [6], while an example of a large project is ER4STEM [17].

However, our context is different from competitions and camps. We are constrained to a two-hour interval. Thus, we adopt a path that includes inquirybased learning (IBL) and problem-based learning (PBL) [24]. We exclude projectbased learning because of the time limitations. But, as explained, we are regularly engaging teachers in high-schools to motivate them to develop project-proposals from extending our activities.

Each activity is, therefore, based on a challenge, accompanied with a lesson plan (enabling different facilitators to conduct the activity) and a booklet of problems. Solving these problems would guide to the solution of the challenges, and we do not expect pupils to solve them during the sessions. The second half of the booklet has some sample solution (not all problems have a unique solution). The booklet is a souvenir for the learners to prompt their teachers or their parents about the solutions, perhaps requesting a further explanation or attempting the problem without knowing an answer and use it for later reflection.

The challenges are, to a certain extent, ill-defined. The first characteristic aspects of our approach that align with IBL is that we expect the participants to play and explore with LEGOß's EV3s and their programming environment (or with SoftBank's Pepper and Nao and the programming environment of Choregraphe) before being introduced to the formal argumentation or the scientific concepts and principles in mathematics and robotics we aim to introduce (the booklet contains a glossary at the end of terminology and nomenclature). Second, our learners are guided to explore, compare, investigate, and repeat their actions in an attempt to discover. Third, we emphasise that "inquiry is the art of questioning and the art of raising questions" [24].

Our presentation of problem-solving follows one of the fundamental strategies for mathematical thinking stated as Polya's First Principle [26]: Understand the problem. Scrutinising the problem by inquiry (looking at simpler cases, exploring the context, and even changing the question ) is part of the learning behaviours and skills we expect to foster.

All of our lessons work for LEGOß's EV3s built under the standard shape for the differential-drive model named TRAC3R (see Figure 1). All programming can be completed using the EV3 kit software (LABVIEW). LEGOß's EV3s are robust, they hardly break, and they represent an affordable choice for many educational institutions, including some parents (even with the separately sold rechargeable pack). LEGO-Mindstorms have been used to stimulate STEM topics for more than 15 years [39], and its different versions have been widely 


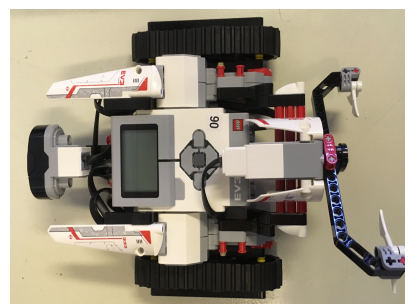

(a) TRAC3R as built

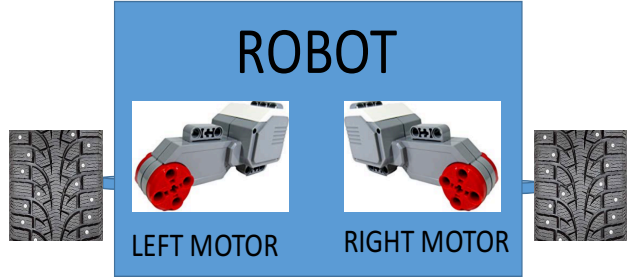

(b) Main components of a differential drive

Fig. 1: The LEGO@'s EV3 TRAC3R standard construction.

used $[7,19,39,29,14,20]$. However, we shift emphasis from the coding skills to the mathematics skills, because we maintain than algorithm understanding (the proof of correctness of an algorithm) is fundamental to relational understanding [34] in mathematics, and central to the quality of software development.

LEGO $®$ produced a series of "EV3 and STEM Curriculum Grids" for different countries, including Australia, where learning objectives of several aspects of the curricula are cross-referenced against LEGO $\AA$ 's activity packs. For example, there is a guide for mathematics curriculum [35]. LEGO $囚$ has also sponsored the First LEGO League $\AA$ and develop the Australian Curriculum Links that cross-reference objective such as "General Capabilities - Literacy", "General Capabilities - Personal and Social Capability", and "General Capabilities - Information and Communication Technology (ICT) Capability" to the opportunities to accommodate such objectives with the involvement with the "First LEGO League" (B). Our activities must inspire learners to seek the reasoning and mathematical problem solving that is developed in high-school and tertiary education. However, our links to mathematical concepts and skills are significantly more specific regarding the Mathematics curriculum by Australian Curriculum, Assessment and Reporting Authority (ACARA). We are inspired by the handson, exploratory and interactive approaches of others [38] for Lower Secondary School, but we offer significantly more complex challenges.

Our approach follows the design of activities inspired by the activities suggested by Mayerová and Veselovská [18]. However, our approach is more into Problem-Based Learning [33] and fostering creativity to apply lateral thinking and cognitive skills that sit high in the Bloom taxonomy. Some of these correspond to so-called 21st-century skills [33]: Critical Thinking Skills, Creativity Skills, Communication Skills, Collaboration Skills. In robotics education, Inquiry-based Learning [29] and Problem-Based Learning [33] have been contrasted with Project-Based Learning [24]. We follow the proposal of Samuels and Poppa [31] for "Themed challenges: Students were briefed on a number of specific challenges around an engaging theme for which they were required to build a robot". Our lessons developed from contact with high-school clubs using robotics for STEM is a similar fashion that theirs [31].

We aim for closer links between mathematical thinking, computational thinking and problem-solving. It is important to analyse the concept or robot, or the 
constructs of programming. But, we seek the fundamental understanding of the notion of algorithm as a generic solution for all the valid inputs. We emphasise the notion of algorithm correctness in the true spirit of relational understanding by Skemp [34]. We share the hope to recognise and guide STEM talent [25]. Most of the topics of our activities seem to be innovative with respect to the multi-grade curriculum of the project READY [28]. Also, there seems to be significant use of the links between educational robotics and topics such as control theory (feedback-loop control and related matters of sensor and actuators) and programming at the university level [36]. Similarly, some service units for educational robotics focused on programming skills and efficacy skills, and cooperative learning![20]. Interest is increased for STEM [20], but the topics remain distant to mathematical concepts. In some cases, the aim for trans-disciplinary curriculum has reached computational thinking, coding, and engineering [11], it still falls short of crossing mathematical thinking and societal impact. We attempt to inspire discussion of technology's impact, but we admit we do not aim to reach the current level of debate [37]. Similarly, we are aware that mathematics provides perhaps the simple models for the physical world, directly propelling the progress of automation and robotics. We use some examples to inspire debate on the successes of artificial intelligence in emulating human cognition [4]; for example, we use Apple's Siri (a voice-controlled personal assistant) to find out who was Winston Churchill, or to convert radians to degrees. These demonstrations of semantic understanding illustrate the impact on recommender systems is no longer merely syntactic matching by search engines and convincingly argues that memorisation and instrumental understanding [34] is becoming less valuable. However, we barely touch on the treatment of values, for example, the potential implementation of systems with emotions [9].

\section{First challenge}

Our first challenge is to program the robotic champion of "paper, scissors, rock". It enables the practice of understanding the problem. This task requires to build a program, so the robot moves its arm in a specific position. This sub-problem is rather simple for the configuration of the robot, as the required program is small: It only requires one block. By using the vast amount of resources about LEGOMindstorms or the guidance of the instructor, we have seen every Year 6 child or older complete this task. Thus, every child succeeds with the first problem. Here is where we bring the other aspects of PBL. Although considered a variant of IBL [24], PBL starts from real and meaningful problems. We link the "paper, Scissors, rock" challenge to the current academic, economic and social debate of ethical decisions by autonomous vehicles. We bring to the attention the reality of moral dilemmas and the suitability of machines selecting the fate of some humans over others. We point to the recent media reports and the controversial and contradictory views different people have on this regard.

Another aspect for adopting a PBL philosophy is that we want to create the need to know about some mathematical tools. The virtue of our problems is that they are easy to formulate, and in principle, they are understood across 
the diversity of the students. However, they are also at the frontier of current research, (recall that the first challenge is an abstraction of the issues around ethical machines). Satisfactory solutions (usually raise more question) can be attempted quite satisfactorily by experimentation. Thus, we immediately suggest creating (coding) behaviour, so the robot can engage, or compete against other similar robots in a tournament of the game of "scissors, paper, rock". Facilitators participate also illustrating the inquiry and problem-solving investigation asking the student question such as "Why?", "How do you know for sure?" "Is that the best possible?" Depending on the progression of the Socratic debate, we have been able to introduce concepts such as Nash equilibrium and mixed strategies. Students have reinforced their notions of probability to calculate the expected wins of the program that chooses randomly among the three options.

While guiding learners for this challenge, we have found opportunities to introduce similar triangles, and as a result, units for bearings, such as degrees and radians. Just debating where is left, right, ahead or behind enables the discussion frames of reference. Converting between polar-coordinates and Cartesian coordinates results in motivation for trigonometric functions (although polarcoordinates has been removed from Math A and Math B in the Queensland curriculum). We can raise questions and inquiry regarding the shapes produced by robotic arms with different joints and introduce motivating discussion for concepts in motion planning such as $C$-space.

There have been learning guides [2] to use robotics and introduce children to programming. Kumar's [2] manual uses the Myro robot to introduce python programming. We allude to Kumar's [2] manual because it shows how natural it is to present the programming of "papers, scissors, rock" with a uniform random choice. It is also interesting that although uniform random choice is the only mixed strategy that is a Nash equilibrium, and in that sense the best, Kumar's [2] manual also shows that if we know biases of the opponent's selection (and humans are creatures full of biases), we can take advantage of such preferences.

We have observed a constructivist revision in most students concept map regarding the notion of machine. The definitions and the popular culture are that once properly set up, machines perform an intended action deterministically. Now we are introducing machines we cannot predict what they will do (recall, the champion robot of "papers, scissors, rock" must be unpredictable).

\section{Second Challenge}

Our second challenge is simply stated.

Construct a program that receives two inputs to travel in the same circle but different speed. Explain (justify) why it works correctly.

This challenge favours PBL over IBL, because the emphasis shifts slightly from inquiry skills to problem-solving skills, and in particular reasoning strategies. But our intention is to fulfil Bell's first principle of Connectedness [5]. We expect that each new idea is linked to an old one enabling students to progress to a common understanding. We aim to guide them to produce a kinematic 
model for the differential drive. This is a challenging task. Depending on several assumption regarding the nature of the motors, it sits at the frontier of some current investigations (the derivation of the classical kinematic model for a differential robot is sometimes explained with not much notation [32, Page 61-62] and rapidly [27]). However, sometimes, much more detailed explanations are provided [30, Section 2.4]. Kinematics models only some aspects of the situation. Thus, the nature of the potential open avenues to the question, or the opportunity for the pupils to inquiry about the assumptions for an acceptable solution (typical assumptions are suitable for kinematic modelling: the robot is on a rigid surface and not or sand or on a slippery surface, and learners adopted them the context of the room where we carry out the activities).

The analogies with physical situations (role-playing situation of pushing with only one motor, or both at the same speed) and producing drawings should provide structure and context (as per Bell's second principle [5]). But as before, we let the learners explore. If they are able to discuss with others we have found that the typical solution that emerges is one where the differential robot has one motor with no power, and the other motor uses the input value to set the speed. This is a correct solution, but it is a special case. Some student will produce some other extreme special cases. For example, one motor with the inverse (negative) speed as the other motor and using the input to regulate the magnitude of the speed. Under this conditions, the robot will spin on its centre, and at different speeds, and some students will argue that this is a very small circle. The exploration by the students take several directions, it is unpredictable. But this indeed how mathematics is constructed. Originally, the kinematic model of a differential drive was not found in a book, it was constructed. Building a program so that a differential robot travels the same circle but at different speed is relatively easy if one is familiar or has a description of the kinematic model for such robot. The challenge is to keep the radius constant although the speeds in the left and right wheels are different. This concept already can establish links to some mathematical thinking, in particular, to the notion of curvature.

We do not claim all students from Year 6 to Year 12 reach all the steps, of the intend reasoning path, but we have observer Year 6 pupils discover the entire argument, and we have observed Year 12 learners failing to complete it, but at least all have gained an appreciating for the reasoning behind the mathematical development. This is precisely what PBL proponents suggest [24]: to identify sub-problems, activating prior knowledge, build models, specify and elaborate on knowledge, and derive justifiable conclusions.

Some of the explorations by students may lead them to the first observation: as long as the speed of both motors does not change (and is somewhat not too large), then the differential drive result is some circle. They probably will discover that the difference between the speed of the right wheel and the left wheel is a significant factor in regulating the diameter of the circle. Playing with different program usually inspires them to notice that very high speeds may cause undesirable effects, like the robot skidding and producing what seems random (or at least hard to predict) trajectories. Some of these examples are 


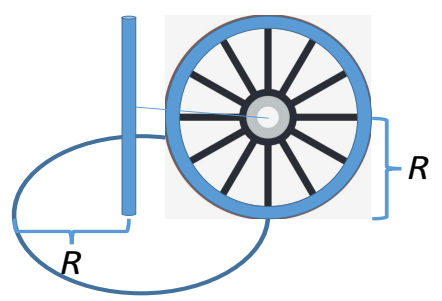

(a) Radius of wheel is same as radius of circular trajectory

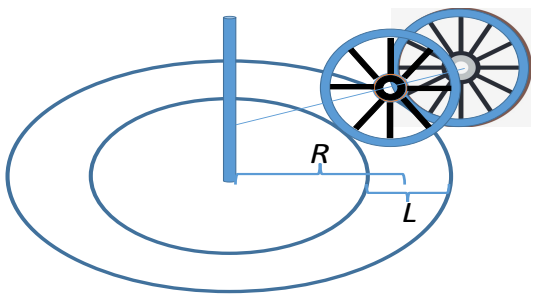

(b) If two wheels complete laps in the same time, the outer spins faster

Fig. 2: Figures that assist the argument to build a kinematic model of a differential-drive.

special solution that correspond to Polya's principle to explore special cases [26]. We can usually guide them to the discovery that the magnitude of the difference in speed contributes to the radius of the circle's trajectory. Moreover, we find again another special case, when the magnitude and direction of the speed of both motors is the same, the robot travels in a straight line. This allows us to suggest mathematical abstractions of geometry where the straight line is a special circle with its centre at infinity (note the link with the notion of limit).

As an aside, because the schedule for the activity does not allow, we consider in the booklet (and in presentations to high-school teachers of the activities to build lessons) some other examples to motivate exploring kinematics (as mathematics of motion without considering the forces that affect the motion). At least we hope teachers can suggest to learners that there is much to contribute in solving problems in robotics considering other wheel arrangements, familiar to students, such as a bicycle (equivalently a motorbike or scooter). We find that some concepts are within the intuition of students, for example, the notion of instantaneous centre of curvature.

But, for the allocated time, our activity plan motivates learners to follow the experimentation with working on reasoning with drawings. Producing drawings is also part of the problem-solving strategies by Polya [26] who suggest to draw pictures. But such drawings already consist of an abstraction, that removes what the problem-solver considers not essential. Those sketches are now models. A crucial model that emerges in some students is derived of the special case of considering just one wheel (like a mono-cycle). What trajectory is performed by a mono-cycle if the centre of the wheel is attached by a rigid string to a post, and the string slides (it does not wrap around the post)?

We inspire learners with some simple cases (Polya's principles once more): what if the string's length is as long as the radius of the wheel (refer to Figure 2a)? This special case represents the wheel rotating on another wheel and can be illustrated physically with LEGO's gears. How many rotations will the wheel do over a circular trajectory that has double the radius? What if the trajectory is a circle with $k$-times the radius? We are aiming at learners discovering the meaning behind a formula that relates the linear speed of the wheel with the angular speed around the trajectory of the circle. Recall that our challenge is to 
identify how to command with software the wheels of a differential-drive robot around a circle with fixed radius (but to regulate the speed).

Typically students discover by experimentation that if they double (or multiply by the same constant) the magnitude of the speeds for both motors, the robot travels the same circle. What raises the bar about this exercise is the justification. The aim here is to reach relational understanding of mathematics as proposed by Skemp [34]. Relational understanding is equivalent, in our view, to understanding why an algorithm works. Instrumental understanding is equivalent to being able to execute the algorithm. Naturally, to inspire students into studying algorithms, we feel the relational understanding [34] of mathematics and computer science is of primary importance.

If another wheel rotated around the post further away (on a larger circle), such second wheel would spin faster (Figure $2 \mathrm{~b}$ ), why? But as long as they go around the post at constant speed, they each rotate at their individual constant speed. Does the separation of the wheels play a role? Bringing the recently build knowledge assist with this. The physical robot is hard to modify to explore enlarging/reducing the separation $L$ between the wheels, but the graphical model should enable student to discover that the further apart the second wheel is, the faster it must spin to circumvent the post. Again, most of the debate should be prompted encouraging and fostering reflection and critical thinking: alternating from Why? to What if? and attempting to re-formulate the problem into other problems. As part of our IBL approach, and since Polya [26], some of the elements of problem-solving consist of reformulating the question.

The next step guides learners to the observation that there are three circles, when the robot travels at constant speed over a circle. There is the circular trajectory of the left wheel, there is the circular trajectory of the right wheel and there is the circular trajectory of the centre of the axel that connects the wheels (and that we may chose that point as the origin for our frame of reference for the robot). When the robot travels at constant speed the angular velocity around the instantaneous centre of curvature is the same for these three circles. We may not use the term angular velocity, (but from the guided analysis, and in particular Polya's principle of working in the one understanding the question as a problem-solving strategy), the challenge statement is translated into going around in a circle at a controllable number of laps per unit of time.

We can proceed to some mathematical encoding, and move from the geometric model to the mathematical model. Again, we have found that the ability from students to reach these stages is independent of their schooling year classification. Although some familiarity with algebra and in manipulating linear equations does enable some students to build the argument themselves. If the radius of the circular trajectory is $R$ and the separation between the wheels (the length of the axle in the robot is $L$, then fact that the rigid robot is travelling at constant speed means that the angular velocity of the wheels in the environment is the same. Depending on the frame of reference, whether the robot is drawn travelling clockwise or counter-clockwise (for our argument refer to Figure 2b), we may get some symmetrical argument, but for the circles of the wheels: 


$$
\text { angular velocity }=V_{r} /\left(R+\frac{L}{2}\right) \quad \text { and } \quad \text { angular velocity }=V_{l} /\left(R-\frac{L}{2}\right)
$$

where $V_{r}$ is the linear velocity of the right wheel, and $V_{l}$ is the linear velocity of the left wheel. Some learners could encode the situation as

$$
V_{r}=\text { angular velocity } \times\left(R+\frac{L}{2}\right) \quad \text { (1) } V_{l}=\text { angular velocity } \times\left(R-\frac{L}{2}\right)
$$

already because of the earlier exploration of the single wheel on a circular trajectory which provides a formula of the form linear velocity $=R \times$ angular velocity where $R$ is the radius of the trajectory.

Subtracting Equation (2) from Equation (1) gives $V_{r}-V_{l}=L \times$ angular velocity or equivalently

$$
\text { angular velocity }=\left(V_{r}-V_{l}\right) / L \text {. }
$$

This equation should be a mathematical model that represent all the initial hands-on exploration with the robot. Namely, it does represent that when both linear speeds are the same there is no angular velocity, the robot moves on a straight line! Moreover, if we make $V_{r}, V_{l}$ of equal magnitude and opposite sign, then the robot spins on itself, with angular velocity proportional to the magnitude $\left|V_{r}\right|=\left|V_{l}\right|$.

This first part of the kinematic model describes how the orientation of the robot (what is it looking at straight ahead, or it bearing) changes as the software controls each motor speed (typically power and direction, already suggesting the notion of vector as magnitude and orientation). However, the challenge is to set the radius $R$. However, now adding Equation (2) and Equation (1), and substituting the angular velocity given by Equation $(3)$ gives $R=\frac{L}{2} \frac{\left(V_{l}+V_{r}\right)}{\left(V_{l}-V_{r}\right)}$. Now is clear that if the new speeds are $V_{l}^{\prime}=k V_{l}$ and $V_{l}^{\prime}=k V_{r}$ the radius is the same, and Equation (3) shows the angular velocity is not the same.

The previous argument is also the kinematic justification of why a robot with a differential-drive performs circles. While many kinematics concepts have traditionally been regarded as topics suitable for advanced undergraduates and graduate students $[10,32]$, we argue here that the hands-on activity enables even Year-6 learners to gain significant understanding of the techniques and an appreciation for the usefulness of mathematical thinking.

\section{Third challenge}

We introduce this challenge reviewing effects of the industrial revolution, the birth of engineering and wide-spread automation. We discuss the video Humans Need Not Apply (https://www.youtube.com/watch?v=7Pq-S557XQU) as it is thought-provoking because it suggest automation will move not only from replacing people for physical tasks, but also for intellectual tasks.

Our third challenge is an introduction to feedback-control systems. Although our pupils rarely have any exposure to calculus, they can be exposed to the notion of a feedback-loop by requesting their robot to follow a line or to maintain 
a distance to another moving robot. Actually, it is quite intuitive that the software must do something every time it detects an error in such regularisation challenges. We have no space to describe the plan for the activity, but suffice to say that rather than using the approach of control theory (that studies the proportional-derivative-integral (PID) controller using the tools from calculus and maybe then the tools of transforms to build the discrete PID), we motivate directly discrete PIDs. This turns our to be quite natural, since discrete PIDs are the only type that can be used with the software and the robots.

\section{Conclusion}

We have also described the activities to 3 groups of high-school teachers as part of two professional-development conferences to teachers. We performed a survey with the school teachers [13]. The survey consisted of 8 question in a 5 -point Likert scale and was completed anonymously by 38 participants. The preferred response to all questions is highly indicative that the activities could be incorporated in high-school lessons and would foster the interest in learners for problem-solving using mathematical tools. For all questions, at least $84 \%$ of the responses were the extreme positive response. Moreover, never an educator

chose the extremely negative response. For all questions, never more than 2 answers were not positive.

\section{References}

1. Åström, K.J., Murray, R.M.: Feedback Systems - An Introduction for Scientists and Engineers, second edn. Princeton University Press (2018)

2. Axelrod, B., Balch, T., Blank, D., Eilbert, N., Gavin, A., Gupta, G., Gupta, M., Guzdial, M., Jackson, J., Johnson, B., Kumar, D., Muhammad, M.N., O'Hara, K., Prashad, S., Roberts, R., Summet, J., Sweat, M., Tansley, S., Walker, D.: Learning computing with robots. IPRE Institute For Personal Robots in Education (2008). URL www.roboteducation.org

3. Barrington, F., Evans, M.: Year-12 mathematics participation in Australia. Australian Mathematical Sciences Institute (2017)

4. Beddoes, Z.M. (ed.): Special Report on Artificial Intelligence, 5, vol. 4. The economist, The address of the publisher (2016)

5. Bell, A.: Principles for the design of teaching. Educational Studies in Mathematics 24(1), 5-34 (1993)

6. Christofi, N., Talevi, M., Holt, J., Wormnes, K., Paraskevas, I.S., Papadopoulos, E.G.: Orbital robotics: A new frontier in education. In: 6th Int. Conf. on Robotics in Education, pp. 20-26. Roboptics (2015)

7. Chung, C.J., Cartwright, C., Cole, M.: Assessing the impact of an autonomous robotics competition for STEM education. Journal of STEM Education 15(2), 24-34 (2014)

8. Dessimoz, J.: International robotics competitions as excellent training grounds for technical education and student exchanges. In: 6th Int. Conf. on Robotics in Education, pp. 86-93. Roboptics (2015)

9. Dessimoz, J.: Natural emotions as evidence of continuous assessment of values, threats and opportunities in humans, and implementation of these processes in robots and other machines. In: M. Bhatt, A. Lieto (eds.) 1st Int. Workshop on 
Cognition and Artificial Intelligence for Human-Centred Design 2017 co-located with IJCAI, CEUR Workshop Proc., vol. 2099, pp. 47-55. CEUR-WS.org (2018)

10. Dudek, G., Jenkin, M.: Computational Principles of Mobile Robotics, 2nd edn. Cambridge University Press, New York, NY, USA (2010)

11. Eguchi, A.: Robotics as a learning tool for educational transformation. In: 4th Int. Workshop Teaching Robotics, Teaching with Robotics \& 5th Int. Conf. Robotics in Education, pp. 27-34 (2014)

12. Eguchi, A.: RoboCupJunior for promoting STEM education, 21st century skills, and technological advancement through robotics competition. Robotics and $\mathrm{Au}-$ tonomous Systems 75, 692 - 699 (2016). DOI doi.org/10.1016/j.robot.2015.05.013

13. Estivill-Castro, V.: Linking mathematics curriculum and concepts to robotics activities. In: INTED2019 Proceedings, 13th Int. Technology, Education and Development Conference, pp. 8012-8017. International Academy of Technology, Education and Development, IATED (2019)

14. Fagin, B., Merkle, L.: Measuring the effectiveness of robots in teaching computer science. SIGCSE Bull. 35(1), 307-311 (2003). DOI 10.1145/792548.611994

15. Flexner, A.: The Usefulness of Useless Knowledge. Princeton University Press, Princeton (2017)

16. Kandlhofer, M., Steinbauer, G., Sundström, P., Weiss, A.: Evaluating the longterm impact of RoboCupJunior: A first investigation. In: D. Obdržálek (ed.) 3rd Int. Conf. on Robotics in Education, pp. 87-94. MATFYZPRESS (2012)

17. Lammer, L., Lepuschitz, W., Kynigos, C., Giuliano, A., Girvan, C.: ER4STEM educational robotics for science, technology, engineering and mathematics. In: Robotics in Education, Advances in Intelligent Systems and Computing, vol. 457, pp. 95-101. Springer International Publishing, Cham (2017). DOI doi.org/10.1007/ 978-3-319-42975-5\_9

18. Mayerová, K., Veselovská, M.: How we did introductory lessons about robot. In: 4th Int. Workshop Teaching Robotics, Teaching with Robotics \& 5th Int. Conf. Robotics in Education, pp. 127-134 (2014)

19. Mohr-Schroeder, M.J., Jackson, C., Miller, M., Walcott, B., Little, D.L., Speler, L., Schooler, W., Schroeder, D.C.: Developing middle school students' interests in STEM via summer learning experiences: See Blue STEM camp. School Science and Mathematics 114(6), 291-301 (2014). DOI 10.1111/ssm.12079

20. Mosley, P., Ardito, G., Scollins, L.: Robotic cooperative learning promotes student STEM interest. American Journal of Engineering Education 7(2), 117-128 (2016)

21. Nugent, G., Barker, B., Grandgenett, N.: The effect of 4-H robotics and geospatial technologies on science, technology, engineering, and mathematics learning and attitudes. In: J. Luca, E.R. Weippl (eds.) EdMedia + Innovate Learning 2008, pp. 447-452. Association for the Advancement of Computing in Education (AACE), Vienna, Austria (2008)

22. Nugent, G., Barker, B., Grandgenett, N., Welch, G.: Robotics camps, clubs, and competitions: Results from a U.S. robotics project. In: 4th Int. Workshop Teaching Robotics, Teaching with Robotics \& 5th Int. Conf. Robotics in Education, pp. 1118 (2014)

23. Nugent, G.C., Barker, B., Grandgenett, N.: The impact of educational robotics on student STEM learning, attitudes, and workplace skills. In: Robotics: Concepts, Methodologies, Tools, and Applications, p. 1442-1459. Information Resources Management Association, IGI Global, Hershey, PA (2012). DOI 10.4018/ 978-1-4666-4607-0.ch070 
24. Oguz-Unver, A., Arabacioglu, S.: A comparison of inquiry-based learning (IBL), problem-based learning (PBL) and project-based learning (PJBL) in science education. Academia Journal of Educational Research 2(7), 120-128 (2014). DOI dx.doi.org/10.15413/ajer.2014.0129

25. Pittí, K., Curto, B., Moreno, V., Ontiyuelo, R.: CITA: : Promoting technological talent through robotics. In: D. Obdržálek (ed.) 3rd Int, Conf. on Robotics in Education, pp. 113-120. MATFYZPRESS (2012)

26. Polya, G.: How to Solve It: A New Aspect of Mathematical Method, second edn. Princeton University Press (1957)

27. Robins, M., Somashekhar, S.H.: Trajectory tracking and control of differential drive robot for predefined regular geometrical path. Procedia Technology 25, 1273 1280 (2016). DOI doi.org/10.1016/j.protcy.2016.08.221. 1st Global Colloquium on Recent Advancements and Effectual Researches in Engineering, Science and Technology - RAEREST 2016 on April 22nd \& 23rd April 2016

28. Rubenzer, S., Richter, G., Hoffmann, A.: Development of a multi-grade curriculum: Project "READY". In: 6th Int. Conf. on Robotics in Education, pp. 60-65. Roboptics (2015)

29. Rursch, J.A., Luse, A., Jacobson, D.: IT-Adventures: A program to spark IT interest in high school students using inquiry-based learning with cyber defense, game design, and robotics. IEEE Transactions on Education 53(1), 71-79 (2010). DOI 10.1109/TE.2009.2024080

30. Salem, F.A.: Dynamic and kinematic models and control for differential drive mobile robots. Int. J. of Current Engineering and Technology 3(2), 253-263 (2013)

31. Samuels, P., Poppa, S.: Developing extended real and virtual robotics enhancement classes with years 10-13. In: Robotics in Education, Advances in Intelligent Systems and Computing, vol. 457, pp. 69-81. Springer International Publishing, Cham (2017). DOI 10.1007/978-3-319-42975-5 \-7

32. Siegwart, R., Nourbakhsh, I.R., Scaramuzza, D.: Introduction to Autonomous Mobile Robots, 2nd edn. The MIT Press (2011)

33. Sierra Rativa, A.: How can we teach educational robotics to foster 21st learning skills through PBL, Arduino and S4A? In: Robotics in Education, pp. 149-161. Springer International Publishing, Cham (2018). DOI doi.org/10.1007/ 978-3-319-97085-1 \_15

34. Skemp, R.: Relational understanding and instrumental understanding. Mathematics Teaching 77, 20-26 (1976)

35. Stanley, K.: Learning with LEGOßeducation: How robotics can meet the NSW technology mandatory 7-8 syllabus outcomes. LEGO Education

36. Swenson, J.: Examining the experiences of upper level college students in 'introduction to robotics'. In: 6th Int. Conf. on Robotics in Education, pp. 72-77. Roboptics (2015)

37. Turkle, S. (ed.): Reclaiming Conversation: The Power of Talk in a Digital Age. Penguin Press, New York, NY, USA (2015)

38. Veselovská, M., Mayerová, K.: LEGO WeDo curriculum for lower secondary school. In: Robotics in Education, pp. 53-64. Springer International Publishing, Cham (2018). DOI doi.org/10.1007/978-3-319-62875-2\_5

39. Whitman, L.E., Witherspoon, T.L.: Using LEGOS to interest high school students and improve K12 STEM education. In: 33rd Annual Frontiers in Education, 2003. FIE 2003., vol. 2, pp. F3A_6-F3A_10 (2003). DOI 10.1109/FIE.2003.1264721

40. Wu, H.: Basic skills versus conceptual understanding: A bogus dichotomy in mathematics education. American Educator 23(3), 1-7 (1999) 